\title{
The propagation of longitudinal stress waves in an infinite rod with a viscoelastic region
}

\author{
Aleksei Pashkov ${ }^{1, *}$ \\ ${ }^{1}$ National Research Moscow State Building University, 129337, Yaroslavskoe shosse, 26, Moscow, \\ Russia
}

\begin{abstract}
The problem of propagation of longitudinal stress waves in an infinite piece-wise homogeneous rod with a viscoelastic region of finite size is solved. A mathematical formulation of the problem and its analytical solution in stresses are obtained. The solution uses the method of the Laplace integral transformation with respect to time. The expressions obtained allow one to determine the stresses in an arbitrary cross-section of the rod at any time. The structure of the solution reflects the process of transformation of the incoming wave with its multiple refraction and reflection at the boundaries of the sections. The solution obtained makes it possible to quantitatively investigate the damping effect of the viscoelastic region and can serve as the basis for selecting the parameters of the insert material when it is used to extinguish the dynamic effects.
\end{abstract}

\section{Introduction}

The use of viscoelastic materials as structural elements, details of mechanisms, and machines is becoming increasingly widespread in such areas as construction (industrial, civil, transportation), engineering, instrumentation, aviation, and many others. This is facilitated, in particular, by the development of technologies for obtaining and practicing the use of polymeric materials, in which the viscoelastic properties are manifested to the greatest extent. Therefore, the study of the behavior of structural elements containing such materials under different types of loading does not cease to be relevant. When it comes to dynamic effects, viscoelastic properties show even those materials that can usually be considered elastic.

A large number of studies have been devoted to the investigation of the behavior of viscoelastic materials and the determination of their properties [1-5]. It is shown that the linear theory of viscoelasticity $[6,7]$, based on the mathematical apparatus of BoltzmannVolter, can be applied to most polymers with sufficient accuracy.

Problems connected with wave processes in rod viscoelastic elements are solved in works [8-13]. We also mention works in which the variability of the cross-section of the rods is taken into account [14], as well as heterogeneity [15], anisotropy [16].

In [17], the problem of the propagation of a shock pulse in a semi-infinite rod consisting of two sections is solved, one of which (finite) has viscoelastic properties.

\footnotetext{
* Corresponding author: pashkovav@mgsu.ru
} 
In this paper we obtain an analytic solution of the problem of the propagation of stress waves in an infinite rod consisting of three sections. The middle portion serves as a viscoelastic insert between two elastic regions.

\section{Problem Statement}

The material parameters of the two elastic semi-infinite sections of the $\operatorname{rod}(\mathrm{x}<0),(\mathrm{x}>1)$ are assumed to be identical and denoted by the index " 1 "; the parameters of the viscoelastic insert material $0 \leq x \leq l$ are denoted by the index " 2 ."

The longitudinal stress wave $\sigma^{*}(x, t)$, which causes displacement $U^{*}(x, t)$, approaches the boundary $(\mathrm{x}=0)$ along the semi-infinite section of the rod on the left.

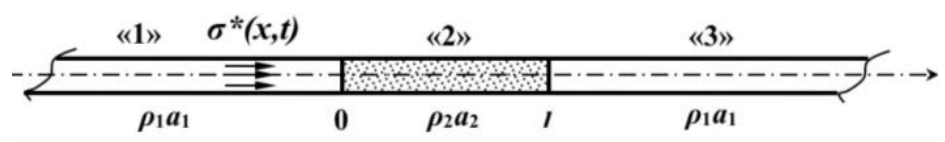

Fig. 1. Infinite rod of constant cross section, having a viscoelastic insert of finite length.

\section{Research Questions}

- How does the process of multiple refraction and reflection of stress waves occur at the boundaries of the rod sections?

- How is the incoming wave transformed as it passes through the viscoelastic region?

\section{Purpose of the Study}

- Obtaining analytical expressions for stresses in an arbitrary cross-section of each of the three sections of the rod.

- Analysis of the solution obtained.

\section{Research Methods}

\subsection{Mathematical formulation of the problem}

Let us first imagine the stress-strain state of the first semi-infinite section $(x<0)$ as a result of superimposing a given wave and waves reflected from the boundaries $(\mathrm{x}<0),(\mathrm{x}>1)$ :

$$
U_{1}(x, t)=U^{*}(x, t)+\tilde{U}(x, t), \quad \sigma_{1}(x, t)=\sigma^{*}(x, t)+\tilde{\sigma}(x, t),
$$

where $\hat{U}(x, t), \hat{\sigma}(x, t)$ is movements and stresses caused by reflected waves. Then the problem of the propagation of waves in sections of the rod reduces to a system of equations 


$$
\begin{array}{ll}
\frac{\partial^{2} \tilde{U}_{1}}{\partial x^{2}}=\frac{1}{a_{1}^{2}} \frac{\partial^{2} \tilde{U}_{1}}{\partial t^{2}} & (x<0) \\
\frac{\partial^{2} U_{2}}{\partial x^{2}}-\int_{0}^{t} f(t-\xi) \frac{\partial^{2} U_{2}}{\partial x^{2}} d \xi=\frac{1}{a_{2}^{2}} \frac{\partial^{2} U_{2}}{\partial t^{2}} & (0 \leq x \leq l) \\
\frac{\partial^{2} U_{3}}{\partial x^{2}}=\frac{1}{a_{1}^{2}} \frac{\partial^{2} U_{3}}{\partial t^{2}} & (x>l)
\end{array}
$$

with boundary conditions

$$
\begin{array}{ll}
x \rightarrow-\infty: & \tilde{U}_{1} \rightarrow 0 \\
x=0: & \tilde{U}_{1}+U^{*}=U_{2}, \quad \tilde{\sigma}_{x x}^{(1)}+\sigma^{*}=\sigma_{x x}^{(2)} \\
x=l: & U_{2}=U_{3}, \quad \sigma_{x x}^{(2)}=\sigma_{x x}^{(3)} \\
x \rightarrow \infty: & \tilde{U}_{1} \rightarrow 0 .
\end{array}
$$

The initial conditions are zero.

The core of the viscoelastic operator has the form [18]:

$$
f(t)=\sum \frac{\gamma_{n}}{\tau_{n}} e t / \tau_{n}
$$

\subsection{Solving the problem in images}

Applying the Laplace integral transformation to the system (1) with respect to $t$ for the transformed displacements

$$
U_{10}=\int_{0}^{t} U_{1} e^{-p t} d t, \quad U_{20}=\int_{0}^{t} U_{2} e^{-p t} d t, \quad U_{30}=\int_{0}^{t} U_{3} e^{-p t} d t
$$

we obtain a system of differential equations

$$
\begin{array}{ll}
\frac{\partial^{2} \tilde{U}_{10}}{\partial x^{2}}=\frac{p^{2}}{a_{1}^{2}} \tilde{U}_{10} & (x<0), \\
\frac{\partial^{2} U_{20}}{\partial x^{2}}=\frac{Q^{2}(p)}{a_{2}^{2}} U_{20} & (0 \leq x \leq l), \\
\frac{\partial^{2} U_{30}}{\partial x^{2}}=\frac{p^{2}}{a_{1}^{2}} U_{30} & (x>l)
\end{array}
$$

with boundary conditions 


$$
\begin{array}{ll}
x \rightarrow-\infty: & \tilde{U}_{10} \rightarrow 0, \\
x=0: & \tilde{U}_{10}=U_{20}, \quad \rho_{1} a_{1}^{2} \frac{d \tilde{U}_{10}}{d x}=\rho_{2} a_{2}^{2} \frac{d U_{20}}{d x}, \\
x=l: & U_{20}=U_{30}, \quad \rho_{2} a_{2}^{2} \frac{d \tilde{U}_{20}}{d x}=\rho_{1} a_{1}^{2} \frac{d U_{30}}{d x}, \\
x \rightarrow \infty: & U_{30} \rightarrow 0,
\end{array}
$$

where $a_{i}=\sqrt{E_{i} / \rho_{i}}$ - wave propagation velocity in the $i$-th part of the rod, $E_{i}, \rho_{i}-$ modulus of elasticity and density of the $i$-th part of the rod, $Q^{2}(p)$ can be approximately represented in the form [19]:

$$
\begin{gathered}
Q^{2}(p)=\frac{p^{2}}{1-f_{0}(p)} \approx\left(p+\frac{c_{1}}{2}\right)^{2}+c_{0}^{2} ; \\
c_{0}^{2}=c_{1}^{2} / 4+c_{2} ; \quad c_{1}=\sum \gamma_{n} / \tau_{n} ; \quad c_{2}=\sum \sum \gamma_{m} \gamma_{j}\left(\tau_{m}^{-1}-\tau_{j}^{-1}\right) .
\end{gathered}
$$

The general solution of system (4) has the form:

$$
\begin{aligned}
& \tilde{U}_{10}=A_{1} e^{-\frac{p}{a_{1}} x}+B_{1} e^{\frac{p}{a_{1}} x} ; \\
& U_{20}=A_{1} e^{-\frac{Q}{a_{2}} x}+B_{2} e^{\frac{Q_{2}}{a_{2}}} ; \\
& U_{30}=A_{3} e^{-\frac{p}{a_{1}} x}+B_{3} e^{\frac{p}{a_{1}} x} .
\end{aligned}
$$

Then for the transformed voltages we obtain expressions:

$$
\begin{aligned}
& \tilde{\sigma}_{x x}^{(1,0)}=\rho_{1} a_{1}^{2} \frac{d \tilde{U}_{10}}{d x}=-\rho_{1} a_{1}^{2} A_{1} e^{-\frac{Q}{a_{1}} x}+\rho_{1} a_{1}^{2} B_{1} e^{\frac{Q}{a_{1}} x} ; \\
& \sigma_{x x}^{(2,0)}=\rho_{2} a_{2}^{2} \frac{d U_{20}}{d x}=-\rho_{2} a_{2}^{2} A_{2} e^{-\frac{Q}{a_{2}} x}+\rho_{2} a_{2}^{2} B_{2} e^{\frac{Q}{a_{2}} x} ; \\
& \sigma_{x x}^{(3,0)}=\rho_{1} a_{1}^{2} \frac{d U_{30}}{d x}=-\rho_{1} a_{1}^{2} A_{3} e^{-\frac{Q}{a_{1}} x}+\rho_{1} a_{1}^{2} B_{3} e^{\frac{Q}{a_{1}} x}
\end{aligned}
$$

Substituting expressions (5), (6) into the boundary conditions (4), we determine the coefficients $A_{i}, B_{i}$ : 


$$
\begin{aligned}
& A_{1}=0 \\
& B_{1}=\frac{S_{1}}{S_{2}} \cdot \frac{\sigma_{0}^{*}(p)}{\rho_{1} a_{1} p^{2}}-\frac{4 \rho_{2} a_{2} Q(p) F_{0}(p)}{S_{2}^{2}} \sum_{m=1}^{\infty}\left(\frac{S_{1}}{S_{2}}\right)^{2 m+1} e^{-\frac{2(m+1) Q}{a_{2}} l} ; \\
& A_{2}=-\frac{2 \sigma_{0}^{*}(p)}{S_{2}} \sum_{m=0}^{\infty}\left(\frac{S_{1}}{S_{2}}\right)^{2 m} e^{-\frac{2 m Q}{a_{2}} l} ; \\
& B_{2}=-\frac{2 \sigma_{0}^{*}(p)}{S_{2}} \sum_{m=0}^{\infty}\left(\frac{S_{1}}{S_{2}}\right)^{2 m+1} e^{-\frac{2(m+1) Q}{a_{2}} l} ; \\
& A_{3}=-\frac{4 \rho_{2} a_{2} Q(p) \sigma_{0}^{*}(p)}{S_{2}^{2}} \sum_{m=1}^{\infty}\left(\frac{S_{1}}{S_{2}}\right)^{2 m} e^{-\frac{(2 m+1) Q}{a_{2}} l+\frac{p}{a_{1}} l} \\
& B_{2}=0,
\end{aligned}
$$

where $S_{1}=\rho_{2} a_{2} p-\rho_{1} a_{1} Q(p), \quad S_{2}=\rho_{2} a_{2} p+\rho_{1} a_{1} Q(p)$.

The relation $S_{1} / S_{2}$ can be represented in the form

$$
\frac{S_{1}}{S_{2}}=-\left\{1+2 \sum_{q}(-1)^{q}\left(\frac{\rho_{1} a_{1}}{\rho_{2} a_{2}}\right)^{q}\left(\frac{p}{Q(p)}\right)^{q}\right\} .
$$

Substituting expressions (7) in the general solution (5), we obtain for the transformed displacements:

$$
\begin{aligned}
& \tilde{U}_{10}=\left(\frac{S_{1}}{S_{2}}\right) \cdot \frac{\sigma_{0}^{*}(p)}{\rho_{1} a_{1} p^{2}} \cdot e^{-\frac{p}{a_{1}} x}-\frac{4 \rho_{2} a_{2} Q F_{0}(p)}{S_{2}^{2}} \sum_{m=1}^{\infty}\left(\frac{S_{1}}{S_{2}}\right)^{2 m+1} \cdot e^{-\frac{2(m+1) Q}{a_{2}} l+\frac{p}{a_{1}} x} \\
& U_{20}=-\frac{2 Q(p) \sigma_{0}^{*}(p)}{S_{2}}\left\{\sum_{m=0}^{\infty}\left(\frac{S_{1}}{S_{2}}\right)^{2 m} \cdot e^{-\frac{Q}{a_{2}}(2 m l+x)}+\sum_{m=0}^{\infty}\left(\frac{S_{1}}{S_{2}}\right)^{2 m+1} \cdot e^{-\frac{Q}{a_{2}}[2(m+1) l-x]}\right\} \\
& U_{30}=-\frac{4 \rho_{2} a_{2} Q(p) \sigma_{0}^{*}(p)}{S_{2}^{2}} \sum_{m=1}^{\infty}\left(\frac{S_{1}}{S_{2}}\right)^{2 m} \cdot e^{-\frac{Q}{a_{2}}(2 m+1) l+\frac{p}{a_{1}}(x-l)} \cdot
\end{aligned}
$$

and for transformed voltages 


$$
\begin{aligned}
& \tilde{\sigma}_{x x}^{(1.0)}=\left(\frac{S_{1}}{S_{2}}\right) \cdot \sigma_{0}^{*}(p) \cdot e^{-\frac{p}{a_{1}} x}-\frac{4 \rho_{1} a_{1} p \rho_{2} a_{2} Q(p) \sigma_{0}^{*}(p)}{S_{2}^{2}} \sum_{m=1}^{\infty}\left(\frac{S_{1}}{S_{2}}\right)^{2 m+1} \cdot e^{-\frac{2(m+1) Q}{a_{2}} l+\frac{p}{a_{1}} x} ; \\
& \sigma_{x x}^{(2.0)}=-\frac{2 \rho_{2} a_{2} Q(p) \sigma_{0}^{*}(p)}{S_{2}} \sum_{m=1}^{\infty}\left(\frac{S_{1}}{S_{2}}\right)^{m} \cdot e^{-\frac{Q}{a_{2}}(2 m l+x)}-\frac{2 \rho_{2} a_{2} Q(p)}{S_{2}} \sum_{m=0}^{\infty}\left(\frac{S_{1}}{S_{2}}\right)^{2 m+1} \cdot e^{-\frac{Q}{a_{2}}[2(m+1) l-x]} ; \\
& \sigma_{x x}^{(3.0)}=-\frac{4 \rho_{1} a_{1} p \rho_{2} a_{2} Q(p) \sigma_{0}^{*}(p)}{S_{2}^{2}} \sum_{m=1}^{\infty}\left(\frac{S_{1}}{S_{2}}\right)^{2 m} \cdot e^{-\frac{Q}{a_{2}}(2 m+1) l+\frac{p}{a_{1}}(x-l)} .
\end{aligned}
$$

\section{3 Finding the originals of stresses}

Turning on $p$ [20] the expressions for the stresses (7) and noticing in advance that for wave processes, i.e. when $p \rightarrow \infty$, the relation $S_{1} / S_{2}$ is close to a constant value

$$
\frac{S_{1}}{S_{2}}=\frac{\rho_{1} a_{1}-\rho_{2} a_{2}}{\rho_{1} a_{1}+\rho_{2} a_{2}}
$$

we obtain expressions for the stresses $\sigma_{x x}^{(i)}$ :

$$
\begin{aligned}
& \tilde{\sigma}_{x x}^{(1)}=\frac{\rho_{2} a_{2}-\rho_{1} a_{1}}{\rho_{2} a_{2}+\rho_{1} a_{1}} \sigma^{*}\left(t-x / a_{1}\right)- \\
& \frac{4 \rho_{1} a_{1} \rho_{2} a_{2}}{\left(\rho_{2} a_{2}+\rho_{1} a_{1}\right)^{2}} \sum_{m=0}^{m_{1}}\left(\frac{\rho_{2} a_{2}-\rho_{1} a_{1}}{\rho_{2} a_{2}+\rho_{1} a_{1}}\right)^{2 m} \cdot\left\{e^{-\alpha s_{1}} \sigma^{*}\left(t-s_{1}-x / a_{1}\right)-\right. \\
& -\int_{t=s_{1}}^{t} \sigma^{*}\left(\xi-\frac{x-l}{a_{1}}\right) \cdot\left[\alpha e^{-\alpha(t-\xi)} I_{0}\left(\alpha \sqrt{(t-\xi)^{2}-s_{1}^{2}}\right)-\alpha^{2} e^{-\alpha(t-\xi)} \frac{I_{1}\left(\alpha \sqrt{(t-\xi)^{2}-s_{1}^{2}}\right)}{\alpha \sqrt{(t-\xi)^{2}-s_{1}^{2}}}(t-\xi)\right] d \xi+ \\
& +\int_{0}^{t}\left\{\left[2 K_{1} \alpha+\left(1-K_{1}^{2}\right) \alpha^{2}(t-\eta)\right] \cdot e^{-K_{2} \alpha(t-\eta)}\left[e^{-\alpha s_{1}} \sigma^{*}\left(t-s_{1}-(x-l) / a_{1}\right)\right]-\right. \\
& \left.-\int_{\eta=s_{0}}^{n} \sigma^{*}\left(\xi-\frac{x-l}{a_{1}}\right)\left[\alpha e^{-\alpha(\eta-\xi)} I_{0}\left(\alpha \sqrt{(\eta-\xi)^{2}-s_{1}^{2}}\right)-\alpha^{2} e^{-\alpha(t-\xi)} \frac{I_{1}\left(\alpha \sqrt{(t-\xi)^{2}-s_{1}^{2}}\right)}{\alpha \sqrt{(t-\xi)^{2}-s_{1}^{2}}}(\eta-\xi)\right] d \xi\right\} d \eta
\end{aligned}
$$




$$
\begin{aligned}
& \sigma_{x x}^{(2)}=\frac{2 \rho_{2} a_{2}}{\rho_{2} a_{2}+\rho_{1} a_{1}} \sum_{m=0}^{m_{1}}\left(\frac{\rho_{2} a_{2}-\rho_{1} a_{1}}{\rho_{2} a_{2}+\rho_{1} a_{1}}\right)^{2 m} \cdot\left\{e^{-\alpha\left(s_{1}+\frac{x}{a_{2}}\right)} \sigma^{*}\left(t-s_{1}-x / a_{2}\right)-\right. \\
& -\int_{t-s_{1}-x / a_{2}}^{t} \sigma^{*}(\xi) \cdot\left[\alpha e^{-\alpha(t-\xi)} I_{0}\left(c_{0} \sqrt{(t-\xi)^{2}-\left(s_{1}+x / a_{2}\right)^{2}}\right)-\right. \\
& \left.-c_{0} e^{-\alpha(t-\xi)} \frac{I_{1}\left(c_{0} \sqrt{(t-\xi)^{2}-\left(s_{1}+x / a_{2}\right)^{2}}\right)}{\alpha \sqrt{(t-\xi)^{2}-\left(s_{1}+x / a_{2}\right)^{2}}}(t-\xi)\right] d \xi+ \\
& +\int_{0}^{t}\left\{\left(2-K_{2} \alpha\right) \cdot e^{-K_{2} \alpha(t-\eta)} \cdot e^{-\alpha\left(\eta-s_{1}-x / a_{2}\right)} \cdot \sigma^{*}\left(\eta-s_{1}-x / a_{2}\right)-\right. \\
& -\int_{\eta-s_{1}-x / a_{2}}^{\eta} \sigma^{*}(\xi) \cdot\left[\alpha e^{-\alpha(\eta-\xi)} I_{0}\left(c_{0} \sqrt{(\eta-\xi)^{2}-\left(s_{1}+x / a_{2}\right)^{2}}\right)-\right. \\
& \left.\left.-c_{0} e^{-\alpha(t-\xi)} \frac{I_{1}\left(c_{0} \sqrt{(\eta-\xi)^{2}-\left(s_{1}+x / a_{2}\right)^{2}}\right)}{\alpha \sqrt{(\eta-\xi)^{2}-\left(s_{1}+x / a_{2}\right)^{2}}}(\eta-\xi)\right] d \xi\right\} d \eta- \\
& -\frac{2 \rho_{2} a_{2}}{\rho_{2} a_{2}+\rho_{1} a_{1}} \sum_{m=0}^{m_{2}}\left(\frac{\rho_{2} a_{2}-\rho_{1} a_{1}}{\rho_{2} a_{2}+\rho_{1} a_{1}}\right)^{2 m+1} \cdot\left\{e^{-\alpha\left(s_{2}-\frac{x}{a_{2}}\right)} \sigma^{*}\left(t-s_{2}-x / a_{2}\right)-\right. \\
& -\int_{t-s_{2}-x / a_{2}}^{t} \sigma^{*}(\xi) \cdot\left[\alpha e^{-\alpha(t-\xi)} I_{0}\left(c_{0} \sqrt{(t-\xi)^{2}-\left(s_{2}-x / a_{2}\right)^{2}}\right)-\right. \\
& \left.-c_{0} e^{-\alpha(t-\xi)} \frac{I_{1}\left(c_{0} \sqrt{(t-\xi)^{2}-\left(s_{2}-x / a_{2}\right)^{2}}\right)}{\alpha \sqrt{(t-\xi)^{2}-\left(s_{2}-x / a_{2}\right)^{2}}}(t-\xi)\right] d \xi+ \\
& +\int_{0}^{t}\left\{\left(2-K_{2} \alpha\right) \cdot e^{-K_{2} \alpha(t-\eta)} \cdot e^{-\alpha\left(s_{2}-x / a_{2}\right)} \cdot \sigma^{*}\left(\eta-s_{2}+x / a_{2}\right)-\right. \\
& -\int_{\eta-s_{2}+x / a_{2}}^{\eta} \sigma^{*}(\xi) \cdot\left[\alpha e^{-\alpha(\eta-\xi)} I_{0}\left(c_{0} \sqrt{(\eta-\xi)^{2}-\left(s_{2}-x / a_{2}\right)^{2}}\right)-\right. \\
& \left.\left.-\alpha^{2} e^{-\alpha(\eta-\xi)} \frac{I_{1}\left(c_{0} \sqrt{(\eta-\xi)^{2}-\left(s_{2}-x / a_{2}\right)^{2}}\right)}{\alpha \sqrt{(\eta-\xi)^{2}-\left(s_{2}-x / a_{2}\right)^{2}}}(\eta-\xi)\right] d \xi\right\} d \eta
\end{aligned}
$$




$$
\begin{aligned}
& \sigma_{x x}^{(3)}=\frac{4 \rho_{1} a_{1} \rho_{2} a_{2}}{\left(\rho_{2} a_{2}+\rho_{1} a_{1}\right)^{2}} \sum_{m=0}^{m_{3}}\left(\frac{\rho_{2} a_{2}-\rho_{1} a_{1}}{\rho_{2} a_{2}+\rho_{1} a_{1}}\right)^{2 m} \cdot\left\{e^{-\alpha s_{3}} \sigma^{*}\left(t-s_{3}-\frac{(x-l)}{a_{1}}\right)-\right. \\
& -\int_{t-s_{3}}^{t} \sigma^{*}\left(\xi-\frac{x-l}{a_{1}}\right) \cdot\left[\alpha e^{-\alpha(t-\xi)} I_{0}\left(c_{0} \sqrt{(t-\xi)^{2}-s_{3}^{2}}\right)-\right. \\
& \left.-c_{0} e^{-\alpha(t-\xi)} \frac{I_{1}\left(c_{0} \sqrt{(t-\xi)^{2}-s_{3}^{2}}\right)}{\alpha \sqrt{(t-\xi)^{2}-s_{3}^{2}}}(t-\xi)\right] d \xi+ \\
& +\int_{0}^{t}\left[2 K_{1} \alpha+\left(1-K_{1}^{2}\right) \alpha^{2}(t-\eta)\right] \cdot e^{-K_{2} \alpha(t-\eta)} \cdot\left[e^{-\alpha s_{3}} \cdot \sigma^{*}\left(\eta-s_{3}-(x-l) / a_{1}\right)-\right. \\
& -\int_{\eta-s_{3}}^{\eta} \sigma^{*}\left(\xi-\frac{x-l}{a_{1}}\right) \cdot\left[\alpha e^{-\alpha(\eta-\xi)} I_{0}\left(c_{0} \sqrt{(\eta-\xi)^{2}-s_{3}^{2}}\right)-\right. \\
& \left.\left.-c_{0} e^{-\alpha(\eta-\xi)} \frac{I_{1}\left(c_{0} \sqrt{(\eta-\xi)^{2}-s_{3}^{2}}\right)}{\alpha \sqrt{(\eta-\xi)^{2}-s_{3}^{2}}}(\eta-\xi)\right] d \xi\right\} d \eta-
\end{aligned}
$$

where $I_{0}(t), I_{1}(t)$ are the modified Bessel functions, $\alpha=c_{1} / 2$,

$$
\begin{aligned}
& s_{0}=s_{2}=2(m+1) l / a_{2}, s_{1}=2 m l / a_{2}, s_{3}=(2 m+1) l / a_{2}, \\
& K_{1}=\frac{\rho_{1} a_{1}}{\rho_{2} a_{2}+\rho_{1} a_{1}}, \quad K_{1}=\frac{\rho_{2} a_{2}}{\rho_{2} a_{2}+\rho_{1} a_{1}} .
\end{aligned}
$$

The upper bounds of the summation $m_{0}, m_{1}, m_{2}, m_{4}$ are determined from the condition that the argument of the function $F(\tau)$ cannot be negative:

$$
m_{0}=\left\{\frac{a_{2}}{2 l}\left(t+\frac{x}{a_{1}}\right)-1\right\}, \quad m_{1}=\left\{\frac{a_{2} t-x}{2 l}\right\}, \quad m_{2}=\left\{\frac{a_{2} t+x}{2 l}-1\right\}, \quad m_{3}=\left\{\frac{a_{2}}{2 l}\left(t-\frac{x}{a_{1}}\right)+\frac{a_{2}-a_{1}}{2 a_{1}}\right\},
$$

$\{\xi\}$ - the integer part of the number $\xi$.

\section{Findings}

- Analytical expressions allowing to calculate the stresses in all three sections of the rod at any time and in an arbitrary section are obtained.

- The solution for each section is the result of multiple refraction and reflection of waves at the boundaries of the sections.

- The viscoelastic insert has a damping effect: with each passage through it, the wavelength increases, and the amplitude decreases. 


\section{Conclusion}

- Expressions for stresses are obtained analytically on the basis of a rigorous mathematical formulation of the problem. These expressions are quite cumbersome for direct application. However, if implemented in the form of a subprogram, it is possible to obtain a convenient tool for the practical study of wave processes in rods with specific material properties.

- The viscoelastic insert can be used as a damping element. In this case, the results obtained can be the basis for selecting its optimal parameters in order to obtain the desired effect.

\section{References}

1. M. L. Vilyams, Rocket Technology and Cosmonautics, 2, 5 (1964)

2. I. I. Goldberg, Mechanical behavior of polymer materials (Khimiya, Moscow, 1970)

3. H. Kolsky, Phil. Mag, 8, I (1956)

4. J. D. Ferry, Viscoelastic properties of polymers (John Wiley \& Sons, New YorkLondon, 1961)

5. M. L. Willams, R. Z. Arenz, Exp. Mech., 4, 9 (1964)

6. R. Christensen, Introduction to the theory of viscoelasticity (Mir, Moscow, 1974)

7. D. Bland, Linear theory of viscoelasticity (Mir, Moscow, 1965)

8. I. G. Philippov, On approximate equations for the vibration of viscoelastic rods (MSU Publishing House, Moscow, 1979)

9. I. G. Filippov, Applied Mechanics, 22, 2 (1986)

10. D. A. Chernous, Mechanics: Scientific Research and Educational-Methodical Developments, 4 (2010)

11. A. I. Zadorozhny, I. A. Bazov, Bulletin of the Rostov State University of Communications, 3, 12 (2003)

12. A. G. Tamrazyan, Herald of the Moscow State University of Economics and Finance, 1, $2(2011)$

13. A. G. Tamrazyan, A. Yu. Stepanov, Izvestia of the Orel State Technical University Series: Building and Transport, 2, 14 (2007)

14. I. G. Filippov, A. V. Dmohovsky, S. A. Skropkin, Proceedings of the Moscow Engineering and Construction Institute, 138 (1977)

15. I. G. Filippov, B. M. Bakhramov, Some problems of the wave dynamics of continuous media and degenerate elastic systems (FAN, Tashkent, 1981)

16. S. G. Lekhnitskiy, Theory of elasticity of an anisotropic body, $2^{\text {nd }}$ edition (Nauka, Moscow, 1977)

17. A. V. Pashkov, Proceedings of Higher Educational Institutions - Textile Industry Technology, 44 (2017)

18. I. G. Filippov, V. G, Cheban, Mathematical theory of oscillations of elastic and viscoelastic plates and rods (Shtiinitsa, Kishenev, 1988)

19. I. G. Filippov, O. A. Egorychev, Wave processes in linear viscoelastic media (Mashinostroyeniye, Moscow, 1983) 
20. G. Baigmen, A. Erdain, Tables of integral transformations (Nauka, Moscow, 1969) 